\title{
Electrical Power Grid Frequency Estimation with Fuzzy Boolean Nets
}

\author{
Nuno M. Rodrigues ${ }^{1}$, Joao P. Carvalho ${ }^{2(\bowtie)}$, Fernando M. Janeiro ${ }^{3}$, \\ and Pedro M. Ramos ${ }^{1}$ \\ 1 Instituto de Telecomunicações, Instituto Superior Técnico, Universidade de Lisboa, \\ Lisbon, Portugal \\ \{nuno.medeiros.rodrigues, pedro.m.ramos\}@tecnico.ulisboa.pt \\ 2 INESC-ID, Instituto Superior Técnico, Universidade de Lisboa, Lisbon, Portugal \\ joao.carvalho@inesc-id.pt \\ 3 Instituto de Telecomunicações, Universidade de Évora, Évora, Portugal \\ fmtj@uevora.pt
}

\begin{abstract}
Power quality analysis involves the measurement of quantities that characterize a power supply waveform such as its frequency. The measurement of those quantities are regulated by internationally accepted standards from IEEE or IEC. Monitoring the delivered power quality is even more important due to recent advances in power electronics and also due to the increasing penetration of renewable energies in the electrical power grid. The primary suggested method by IEC to measure the power grid frequency is to count the number of zero crossings in the voltage waveform that occur during $0.2 \mathrm{~s}$. The standard zero crossing method is usually applied to a filtered signal that has a non deterministic and frequency dependent delay. For monitoring the power grid a range between 42.5 and $57.5 \mathrm{~Hz}$ should be considered which means that the filter must be designed in order to attenuate the delay compensation error. Fuzzy Boolean Nets can be considered a neural fuzzy model where the fuzziness is an inherent emerging property that can ignore some outliers acting as a filter. This property can be useful to apply zero crossing without false crossing detection and estimate the real timestamp without the non deterministic delay concern. This paper presents a comparison between the standard frequency estimation, a Goertzel interpolation method, and the standard method applied after a FBN network instead of a filtered signal.
\end{abstract}

Keywords: Power quality · Frequency estimation - Zero crossing · Neural networks $\cdot$ FBN

This work was supported by national funds through FCT, Fundação para a Ciência e a Tecnologia, under project UIDB/50021/2020, PhD program reference $\mathrm{SFRH} / \mathrm{BD} / 130327 / 2017$ and is funded by FCT/MCTES through national funds and when applicable co-funded EU funds under the project UIDB/EEA/50008/2020. 


\section{Introduction}

The quality of the electrical power grid is of utmost importance for normal operation of electrical equipment [1-3]. Due to its importance, power quality has been regulated in various internationally recognized standards. Recent advances in power electronics and the ever increasing penetration of renewable energies that require distributed power converters makes the monitoring of the delivered power quality even more important $[4,5]$. Monitored parameters include the frequency of the supplied power waveform, its RMS amplitude, the existence of harmonics, the effect of noise and the existence of distortions and transients.

Power quality measurements are typically performed only at predefined locations and not on a regular basis, usually to settle disputes between utility companies and consumers. The main reason for this is that commercial power quality analyzers are very accurate, but expensive and bulky. The alternative are smaller analyzers that are less expensive but have worse specifications. Therefore, there is an increasing demand for portable analyzers that can be easily and more universally deployed, with better accuracy than those that are presently available. The advances in the processing power of digital signal processors, available memory, and sensors' availability, have boosted the interest on the development of embedded power quality analyzers that are low-cost, yet very powerful [6].

Frequency estimation and tracking methods are an active research topic in many scientific areas as in power quality assessment [7]. The most basic spectral based methods performs a FFT but to have good spectral resolutions the computational cost increases. There are methods that can improve frequency estimation performing an interpolation with the calculated DFT bins [8],[9]. But for some applications, as in power quality, there is no need to compute a full FFT. Approaches as in [10] that uses Goertzel filters [11] or in [12] use a warped DFT in order to select only a defined spectral area to perform frequency estimation.

For some specific applications power grid frequency must be obtained in a 10 cycle time span, that in a $50 \mathrm{~Hz}$ electrical power grid system corresponds to $0.2 \mathrm{~s}$ period as specified in IEC standard 61000-4-30 [13]. The fundamental frequency is the number of integral cycles counted during the considered time interval, divided by the cumulative duration of those cycles. When using the zero crossing (ZC) method, harmonics, interharmonics and noise should be attenuated to avoid false zero crossings in frequency estimation. A particularly effective solution is to digitally low-pass filter the acquired waveform to attenuate the unwanted effects [14]. A disadvantage of this method is the delay that the filter introduces between the filtered and unfiltered signals. This delay, that is frequency dependent, must be compensated to estimate the real zero crossing timestamp. Another method to perform frequency estimation is based on the Goertzel algorithm with interpolation for frequency estimation. The Goertzel algorithm [11] is an efficient method to estimate individual components of the signal Discrete Fourier Transform (DFT). In order to estimate the power grid frequency, an interpolation algorithm based on the Interpolated Discrete Fourier Transform (IpDFT) of [8] is applied. 
In this paper we study an alternative approach to estimate power grid frequency based on zero crossing detection that uses Fuzzy Boolean Nets (FBN) [15]. Natural or Biological neural systems have a certain number of features that leads to their learning capability when exposed to sets of experiments from the real outside world. They also have the capability to use the learnt knowledge to perform reasoning in an approximate way. FBN are a neural fuzzy model where the fuzziness is an inherent emerging property that were developed with the goal of exhibiting those systems' properties. FBN previous studies have shown promising results in learning and interpolating in applications such as pultrusion wastes [16] or dataflow management systems [17]. In [16] FBN method allows to find the best balance between the material parameters that maximizes the strength of the final composite. In [17] is used to augment performance, rationalization of resources, and task prioritization of dataflows based in probabilistic results of the networks output.

The contents of this paper is as follows: Sect. 2 describes the three different methods in order to perform a frequency estimation; Sect. 3 presents results for simulated data with some PQ events that can affect ZC detection; Sect. 4 compares the results between the three methods from the electrical power grid; In Sect. 5 the conclusions about the comparison of the methods applied.

\section{Frequency Estimation Methods}

\subsection{Filtered Signal with Zero Crossing}

According to IEC 61000-4-30 standard [13], a signal power frequency measure is the number of integral periods divided by the duration of those periods within the considered time interval. This measure can be accomplished by estimating the zero crossing timestamps trough interpolation between the acquired samples. To avoid false zero crossings caused by PQ events, the acquired data should be filtered. The digital filter introduces a delay which offsets the timestamps of the filtered signal zero crossings that is dependent on the signal frequency but can be corrected in order to have a real timestamp [14]. In Fig. 1 an example about the effect of filtering a signal is shown.

\subsection{Goertzel and Interpolation}

The Goertzel algorithm [11] is an efficient method to compute a single DFT tone without having to perform a full FFT. Since the power grid frequency bounds have a limited range, only a few selected spectral components are needed. After the Goertzel components computation, the Interpolated Discrete Fourier Transform (IpDFT) [8] is applied. IpDFT is a spectral based method that estimates the signal frequency based on the calculation of the signal FFT, selecting the highest amplitude spectral component, then its largest neighbour and interpolating them. With Goertzel and IpDFT is possible to achieve an accurate frequency estimation with a lower computational cost. 


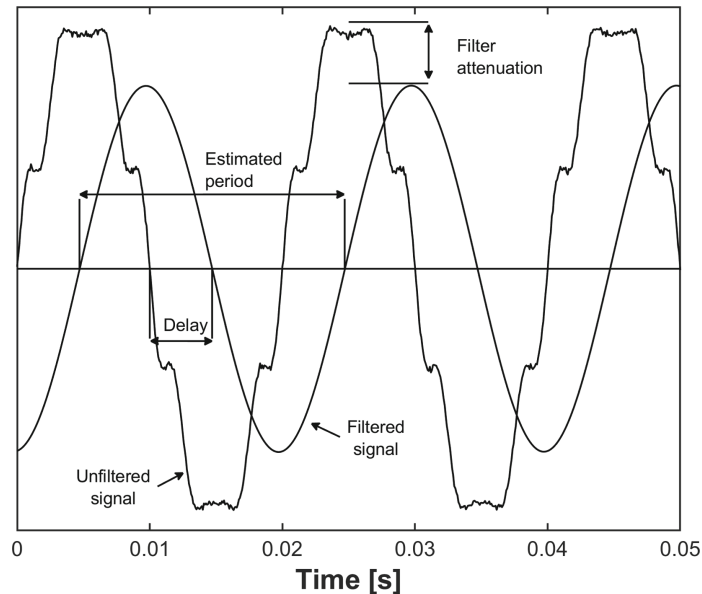

Fig. 1. Example of frequency estimation using digital filtering and zero crossing detection. The input signal is filtered what causes a frequency dependant delay. With a filtered signal is possible to estimate the real zero crossing compensating the delay.

In Fig. 25 DFT bins of a sine signal with spectral leakage are shown. Bold lines represents 5 Goertzel outputs $G_{f}$ that are used to perform a frequency estimation. In this example the higher amplitudes are $G_{50}$ and $G_{55}$ which means that these are the DFT bins to be used in IpDFT algorithm.

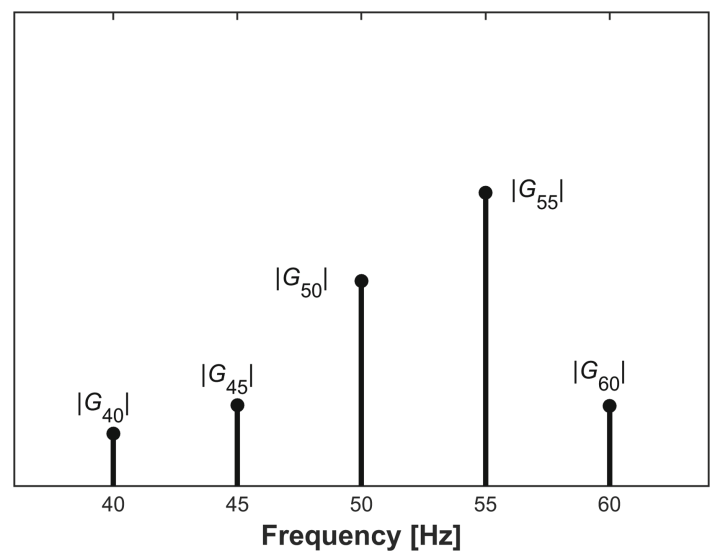

Fig. 2. Example of a signal with spectral leakage where the true frequency is not centered in none of the DFT bins. Interpolation is performed using $G_{50}$ and $G_{55}$ in order to obtain the signal frequency $(53 \mathrm{~Hz})$. 


\subsection{Using FBN to Estimate Power Grid Frequency}

FBN [15] exhibit the natural or biological neural systems features that lead to a learning capability when exposed to sets of experiments from the real world. They also have the capability to use newly gained knowledge to perform approximate qualitative reasoning in the form of "if...then" rules. As in natural systems, FBN are robust and immune to individual neuron or connection errors and present good generalization capabilities that automatically minimize the importance of imbalances and sparseness in the training data. FBN use a Hebbian learning process and are capable of learning and implementing any possible multi-input single-output function of the type: $[0,1]^{n} \times[0,1]$.

FBN consist of neurons that are grouped into areas of concepts (variables). Meshes of weightless connections between antecedent neuron outputs and consequent neuron inputs are used to perform 'if-then' inference between areas. Neurons are binary and the meshes are formed by individual random connections (like in nature). Each neuron comprises $m$ inputs for each antecedent area, and up to $(m+1)^{N}$ internal unitary memories, where $N$ is the number of antecedents. $(m+1)^{N}$ corresponds to maximum granularity. When stimulated, the value of each concept is given by the activated/total neurons ratio. For rules with $N$ antecedents and a single consequent, each neuron has $N \times m$ inputs.

Inference proceeds as follows: The single operation carried out by each neuron is the combinatorial count of activated inputs from every antecedent. For all counting combinations, neurons compare the sampled values with the ones in their unitary memory $(\mathrm{FF})$. If the $\mathrm{FF}$ that corresponds to the sampled value of all antecedents contains the value " 1 ", then the neuron output is also " 1 ". Otherwise, the neuron output is " 0 ". As a result of the inference process (which is parallel), each neuron assumes a boolean value, and the inference result will be given by the neural activation ratio in the consequent area.

Learning is performed by exposing the net to the data input and by modifying the internal binary memories of each consequent neuron according to the activation of the $m$ inputs (per antecedent) and the state of that consequent neuron. Each experiment will set or reset one binary memory of each individual neuron. Due to its probabilistic nature, the FBN must be repeatedly exposed to the same training data for a minimum number of times $(r)$. The optimization of $r$ is not critical since FBN cannot be overtrained. Thus, it is only necessary to guarantee a minimum value that depends on the net parameters $(m, N$, granularity) and sparsity of the training data set.

The idea is to use a 1-input/1-output $\operatorname{FBN}(N=1)$ as a filter without causing a delay. We start by feeding the network with a small set of training points (timestamp/amplitude) sampled during one period; The second step consists in letting the FBN interpolate/estimate the amplitude values for the whole period (based on the few training points), and letting it infer the timestamps of the zero crossing points; Finally we use a standard ZC procedure to estimate frequency. 


\section{Simulated Results}

Different events, such as transients, harmonics or noise can occur in electrical power grids. In this section we present how FBNs behave reagarding $\mathrm{ZC}$ detection when simulating such effects. All tests were performed training and testing the FBN network with 100 repetitions.

\subsection{Cosine}

The first test consists in a fundamental cosine with $0.9 \mathrm{Vpp}$ and $50 \mathrm{~Hz}$. On the left side of Fig. 3 a simulated one period signal is presented. 25 evenly spaced points were automatically selected to train the FBN network (as shown on right side of Fig. 3).
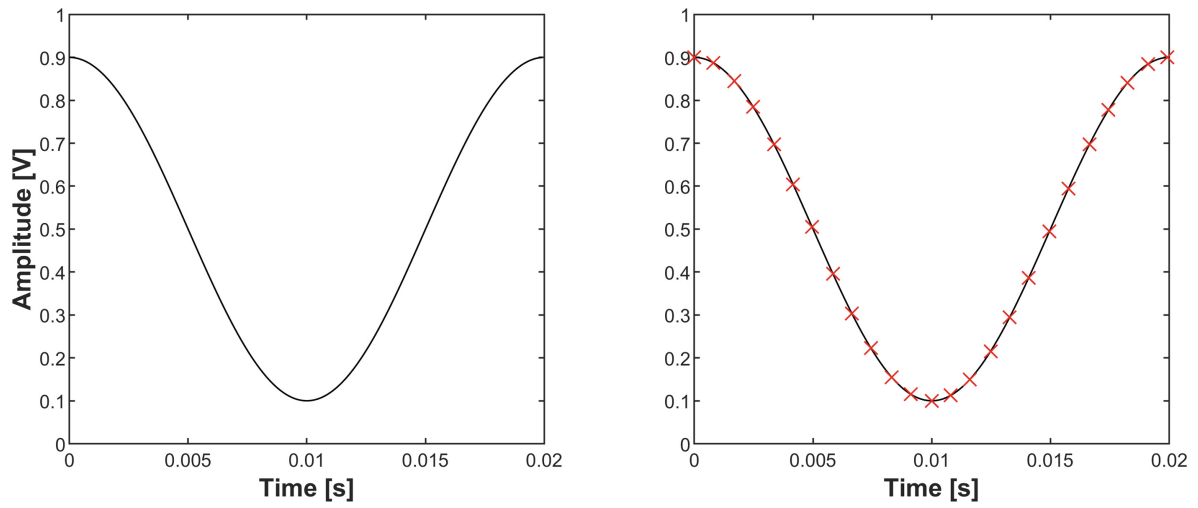

Fig. 3. On the left side a simulated cosine with $0.9 \mathrm{Vpp}$ and $50 \mathrm{~Hz}$ is presented. 25 points were used to train the FBN network.

The system was tested with several parameter configurations, and the best results were obtained using areas with 250 neurons, a sample size $m=60$, and maximum granularity $(m+1)^{N}=61$.

Since the main signal is a cosine, the zero crossings should be detected at $0.005 \mathrm{~s}$ and $0.015 \mathrm{~s}$. The FBN indicated zero crossings at $0.0049 \mathrm{~s}$ and $0.015 \mathrm{~s}$.

\subsection{Sum of Cosines with Same Phase}

The fundamental frequency in electrical power grids is $50 \mathrm{~Hz}$, but harmonics can be present. In this simulation two harmonics $\left(3^{r d}\right.$ and $\left.5^{t h}\right)$ were added. The $3^{\text {rd }}$ harmonic has an amplitude of $5 \%$ of the fundamental signal and the $5^{\text {th }} 6 \% \mathrm{~V}$ that are the maximum amplitudes allowed by the IEEE standard. Figure 4 presents the effects of harmonics in the fundamental signal. Since this frequencies are in phase, zero crossing should not be affected. 


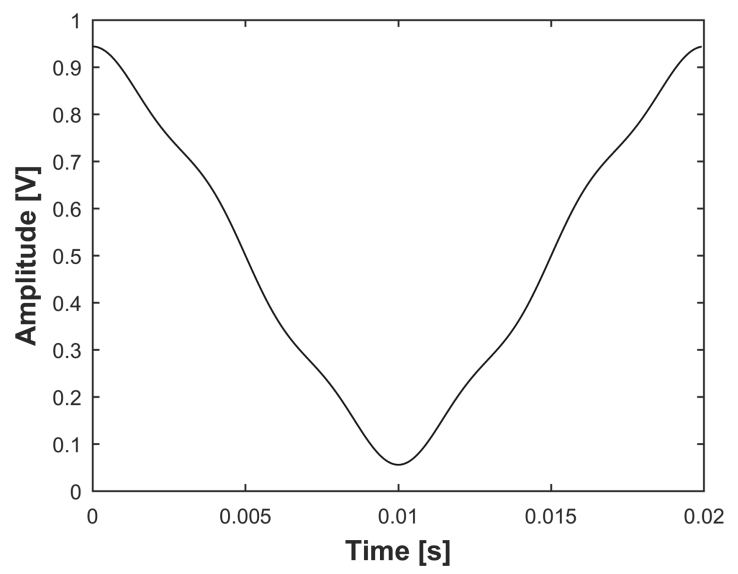

Fig. 4. Representation of a sum of cosines in multiples of fundamental frequency with standard maximum amplitudes.

The zero crossings should still be detected at $0.005 \mathrm{~s}$ and $0.015 \mathrm{~s}$. Using the same configuration as in the previous section, the FBN obtained zero crossings at $0.0045 \mathrm{~s}$ and $0.0151 \mathrm{~s}$.

\subsection{Sum of Cosines with Different Phases}

Harmonics out of phase with the fundamental signal can affect the ZC detection. A filter attenuates higher frequencies, so, the $\mathrm{ZC}$ detection is performed only on the fundamental one. In this case the FBN network will be trained with points that can not represent the fundamental zero cross. In Fig. 5 is presented a sum of signals with different phases and in Fig. 6 the obtained result.

In this test the first crossing was detected at $0.0047 \mathrm{~s}$ and the second one at $0.0150 \mathrm{~s}$.

\subsection{Cosine with Transients}

Transients are another PQ event that could affect the frequency estimation. If a transient occurs without causing a false zero crossing transition, there should not be any problem with frequency estimation. But, as presented in Fig. 7, if a transient occurs near a zero cross transition this event can lead to a false transition getting a wrong frequency estimation.

As is shown in Fig. 8 the training points, represented as red crosses, were affected by the transients. But the FBN network output, represented as the black line, followed the fundamental frequency behavior. This result shows the capability of ignoring some outliers making possible the $\mathrm{ZC}$ detection. In this test the zero crossings were detected at $0.0049 \mathrm{~s}$ and $0.0153 \mathrm{~s}$. 


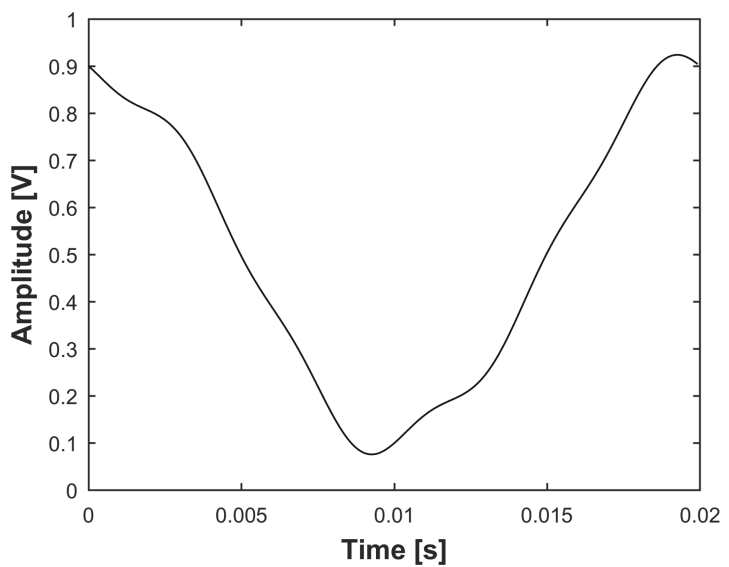

Fig. 5. Representation of a sum of signals with different phases in multiples of fundamental frequency.

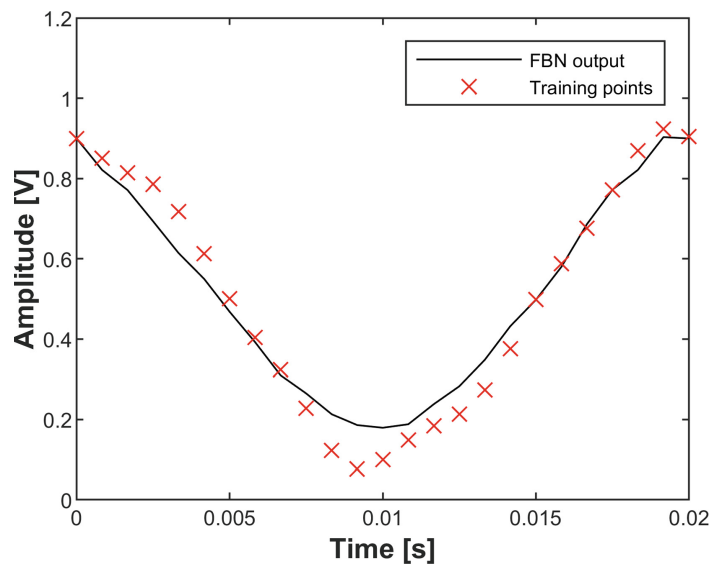

Fig. 6. FBN network output for the situation presented in Fig. 5. FBN output trained with 25 points. Areas of 250 neurons, each neuron performing $m=60$ samples and maximum granularity. Red crosses represents the training points and the black line represents the FBN network output. (Color figure online)

\subsection{Cosine with Noise}

Another event that is common in electrical power grid is the presence of noise. As in the previous test, noise can affect zero crossing detection if occurs near a zero cross transition. This test was performed with a noise of $30 \mathrm{~dB}$ and the signal to estimate its zero crossing is represented in Fig. 9.

In Fig. 10 the output of FBN netowork is shown. Network training points, represented as red crosses, were not following the fundamental signal as it can be seen in the signal minimum peak. But the output of $\mathrm{FBN}$, represented as the 


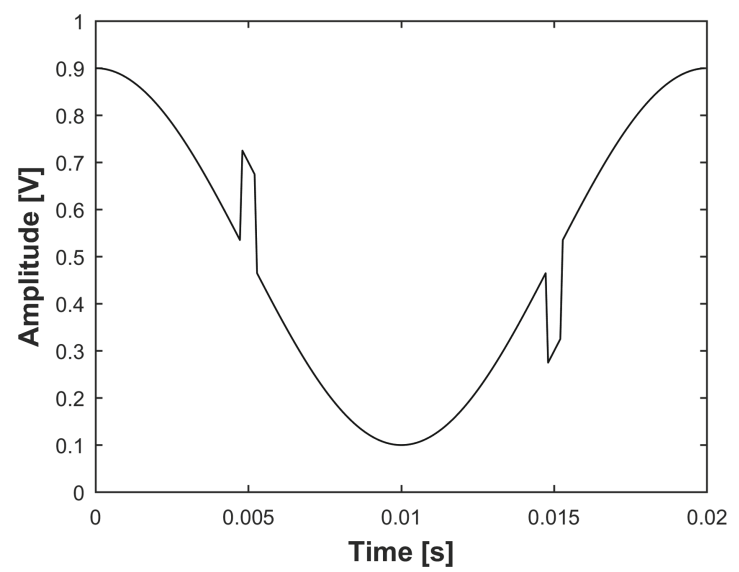

Fig. 7. Representation of transients in a cosine signal on the zero crossings.

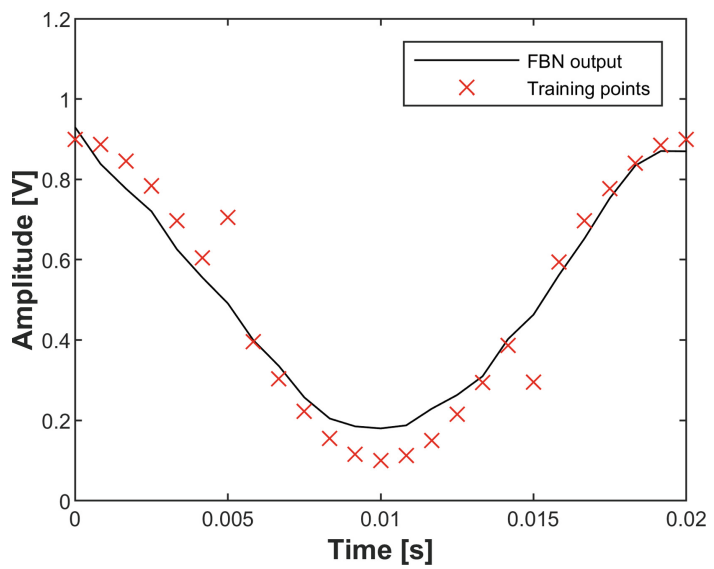

Fig. 8. FBN network output for the situation presented in Fig. 7. FBN output trained with 25 points. Areas of 250 neurons, each neuron performing $m=60$ samples and maximum granularity. Red crosses represents the training points and the black line represents the FBN network output. (Color figure online)

black line, did not follow those transitions. This results shows that the FBN network can attenuate noisy effects. In this simulation zero crossings were detected at $0.0049 \mathrm{~s}$ and $0.0153 \mathrm{~s}$.

\section{Frequency Estimation with Power Grid Dataset}

Once the best FBN parameters for ZC detection were selected (using the examples presented in the previous section), the system was tested on a real dataset. This dataset was acquired in Instituto Superior Técnico - Taguspark with the 


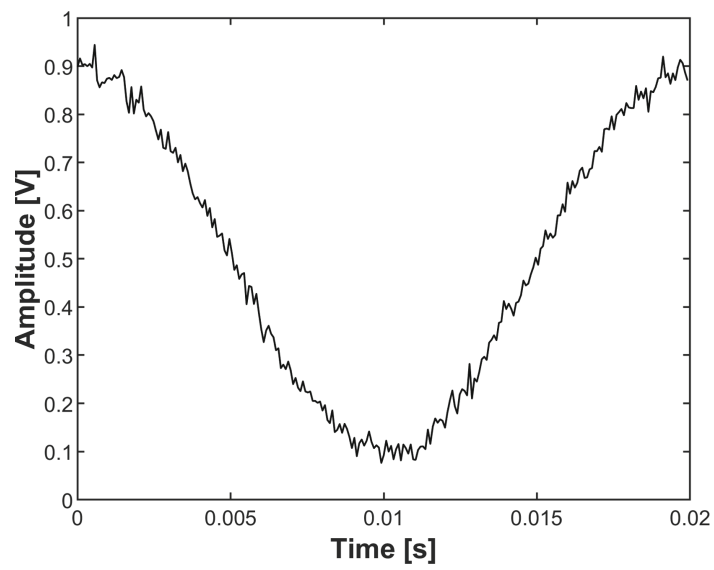

Fig. 9. Representation of a cosine with a measured noise of $30 \mathrm{~dB}$.

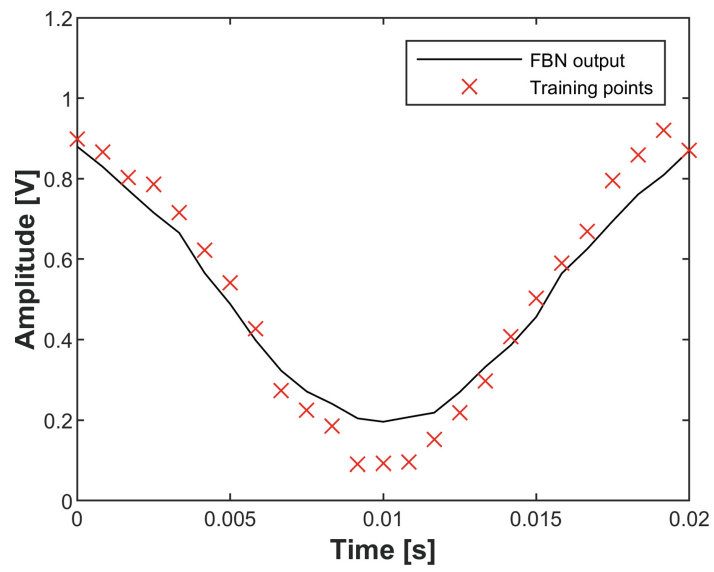

Fig. 10. FBN network output for the situation presented in Fig. 9. FBN output trained with 25 points. Areas of 250 neurons, each neuron performing $m=60$ samples and maximum granularity. Red crosses represents the training points and the black line represents the FBN network output. (Color figure online)

analog input module NI 9215. Data was acquired with a sampling frequency of $12.5 \mathrm{kHz}$, which gives a dataset containing 250 points per period (considering a $50 \mathrm{~Hz}$ frequency). Figure 11 shows an example of a period of a real power grid signal (that corresponds to around $0.02 \mathrm{~s}$ ). In this example it is possible to see some fluctuations in the signal peaks.

As stated in section I, ZC is performed every $200 \mathrm{~ms}$ which translates into 10 periods if a fundamental signal with $50 \mathrm{~Hz}$ is considered. In Fig. 12 the three methods are compared since the beginning of the process until $t=2 \mathrm{~s}$, the equivalent of 10 frequency estimations. "Goertzel with interpolation" starts and ends 


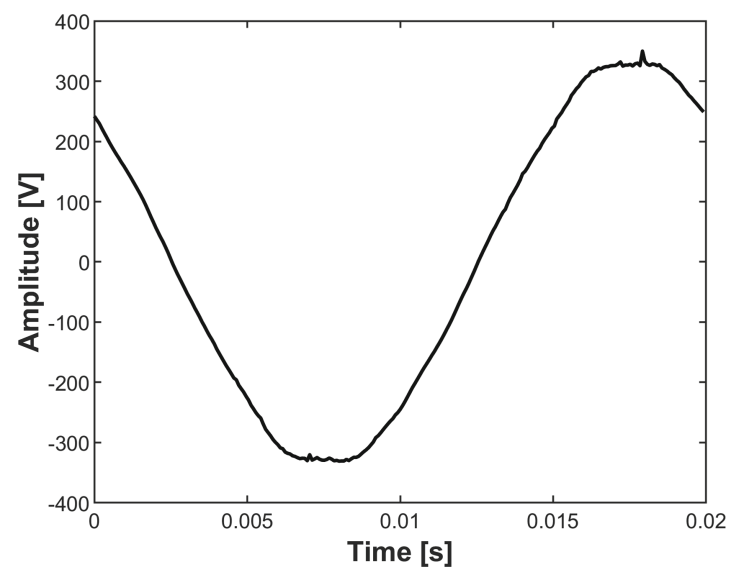

Fig. 11. A period of a real power grid signal acquired with a $12.5 \mathrm{kHz}$ sampling frequency during $0.02 \mathrm{~s}$. In this example some transients occurred near the peaks.

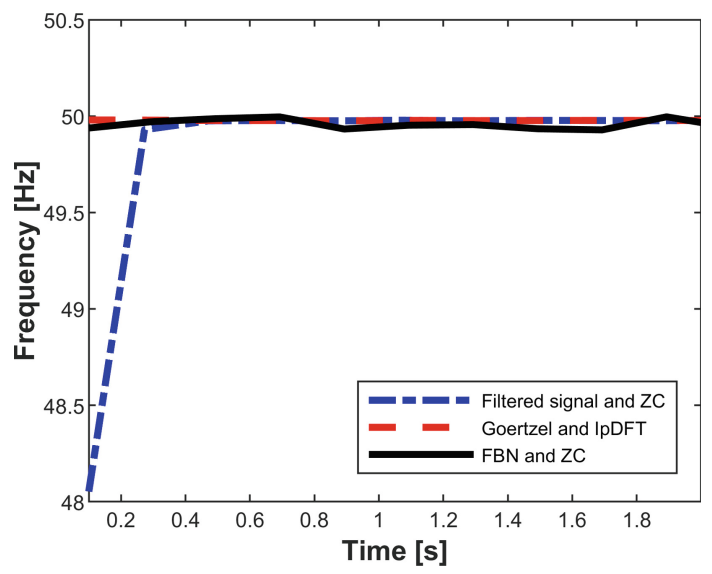

Fig. 12. Frequency estimation along $2 \mathrm{~s}$ in all methods. Digital Filter method starts with a poor estimation due to the time that digital filter takes to establish but then gets a closer estimation to the Goertzel method. FBN with a maximum error of around $0.05 \mathrm{~Hz}$

without visible fluctuations in this scale. The delay in digital filtering method was already compensated in order to have a real timestamp but other effects are shown. Filters need some time before the output is stable, and that is the reason why the filtering method starts with a bad frequency estimation. The FBN method provides good results, but not as precise as any of the other methods. In this segment, the maximum estimation error, when compared with standard and Goertzel methods, was around $0.05 \mathrm{~Hz}$. 


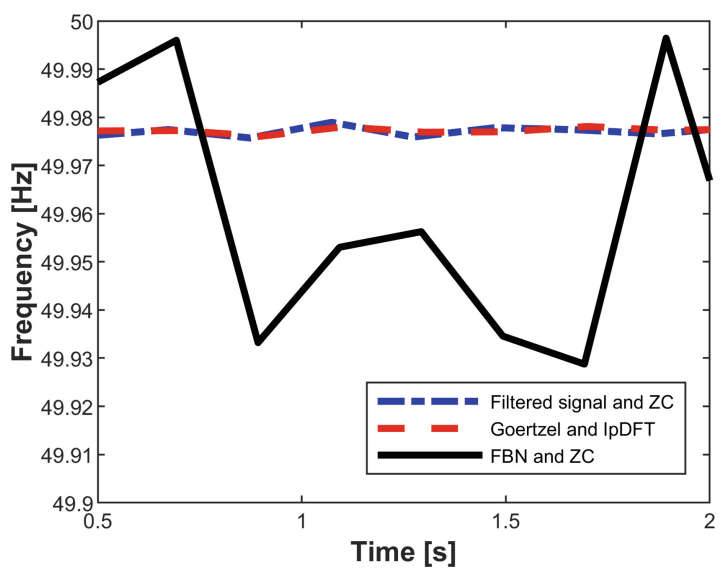

Fig. 13. Zoomed in view: comparison of the three methods during the stable part of the signal.

Figure 13 shows a magnified view of the "stable" part of the signal, where the difference between the three methods is more clear. The estimated frequency is around $49.98 \mathrm{~Hz}$ and, as shown in Fig. 12, FBN method precision is lower than the other methods. Note that the difference is of a very low magnitude, and that this decrease in precision is liked to be compensated when in the presence of events as those exemplified in the previous section.

\section{Conclusions}

In this paper a comparison between three different methods to estimate the power grid frequency is performed in order to study the possibility of using Fuzzy Boolean Networks (FBN) for such application. The two other methods used to validate FBN are the standard power grid frequency estimation which corresponds to counting the number of periods within a given time frame after filtering the input signal, and Goertzel with interpolation.

The main goal of this comparison was to understand the advantages and limitations of using FBN in electrical power grid frequency estimation.

A compromise between time and accuracy was done and better results were obtained training and testing the network 100 times, using 25 training points, an area size of 250 neurons, $m=60$ samples per neuron, and maximum granularity. Comparing with other methods FBN results were marginally worse, but FBN can ignore outliers caused by noise or transients, as shown in Fig. 8 and Fig. 10. In addition, since the network is trained with only 25 points this method could be useful when a low number of measuring points is available. One unavoidable limitation of using FBN is the training and testing time. This makes them unsuitable for real-time applications unless using dedicated hardware. 


\section{References}

1. Arrillaga, J., Watson, N.R., Chen, S.: Power System Quality Assessment, 1st edn. Wiley, Hoboken (2000)

2. Arrillaga, J., Smith, B.C., Watson, N.R., Wood, A.R.: Power System Harmonic Analysis. Wiley, Hoboken (1997). ISBN: 978-0-471-97548-9

3. Fuchs, E.F., Masoum, M.A.S.: Power Quality in Power Systems and Electrical Machines, 1st edn, February 2008. ISBN: 978-0-12-369536-9

4. Guerrero, J.M.: Guest editorial special issue on power quality in smart grids. IEEE Trans. Smart Grid 8(1), 379-381 (2017). https://doi.org/10.1109/TSG.2016. 2634482

5. Montoya, F.G., García-Cruz, A., Montoya, M.G., Agugliaro, F.M.M.: Power quality techniques research worldwide: a review. Renew. Sustain. Energy Rev. 54, 846-856 (2016). https://doi.org/10.1016/j.rser.2015.10.091

6. Ramos, P.M., Radil, T., Janeiro, F.M., Serra, A.C.: DSP based power quality analyzer using new signal processing algorithms for detection and classification of disturbances in a single-phase power system. In: IMEKO TC4, Iasi, Romania, pp. 433-438 (2007)

7. Ramos, P.M., Serra, A.C.: Comparison of frequency estimation algorithms for power quality assessment. Measurement 42(9), 1312-1317 (2009). https://doi.org/ 10.1016/j.measurement.2008.04.013

8. Schoukens, J., Pintelon, R., Van Hamme, H.: The interpolated fast Fourier transform: a comparative study. IEEE Trans. Instr. Measur. 41, 226-232 (1992). https://doi.org/10.1109/19.137352

9. Gong, C., Guo, D., Zhang, B., Aijun, L.: Improved frequency estimation by interpolated DFT method, 29, 4112-4116 (2012). https://doi.org/10.1016/j.proeng.2012. 01.629

10. Chen, W., Dehner, L.G.: A low-cost and high performance solution to frequency estimation for GSM/EDGE. In: Texas Wireless Symposium, pp. 610 (2005). http://citeseerx.ist.psu.edu/viewdoc/download?doi=10.1.1.128.7776\& rep $=$ rep $1 \&$ type $=$ pdf

11. Goertzel, G.: An algorithm for the evaluation of finite trigonometric series. Am. Math. Mon. 65, 34-35 (1958). https://doi.org/10.2307/2310304

12. Franz, S., Mitra, S., Doblinger, G.: Frequency estimation using warped discrete Fourier transform, 83, 1661-1671 (2003). https://doi.org/10.1016/S01651684(03)00079-3

13. IEC 61000-4-30, Electromagnetic compatibility (EMC) - Part 4-30: Testing and measurement techniques - Power quality measurement methods, 3.0 Edition, February 2015

14. Rodrigues, N.M., Janeiro, F.M., Ramos, P.M.: Digital filter performance for zero crossing detection in power quality embedded measurement systems. In: 2018 IEEE International Instrumentation and Measurement Technology Conference (I2MTC), pp. 1-6, May 2018. https://doi.org/10.1109/I2MTC.2018.8409701

15. Tomé, J., Carvalho, J.P.: Fuzzy Boolean nets - a nature inspired model for learning and reasoning. Fuzzy Sets Syst. 253, 1-27 (2014). https://doi.org/10.1016/j.fss. 2014.04.020

16. Castro, A.M., et al.: An integrated recycling approach for GFRP pultrusion wastes: recycling and reuse assessment into new composite materials using Fuzzy Boolean nets. J. Clean. Prod. 66, 420-430 (2014). https://doi.org/10.1016/j.jclepro.2013.10.030

17. Esteves, S., Silva, J.N., Carvalho, J.P., Veiga, L.: Incremental dataflow execution, resource efficiency and probabilistic guarantees with Fuzzy Boolean nets. J. Parallel Distribu. Comput. 79-80, 52-66 (2015). https://doi.org/10.1016/j.jpdc.2015.03.001 